\title{
Design of Strategy Generation System for Urban Comprehensive Disaster Prevention Planning Based on Transfer Bridge
}

\author{
Guannan Fu*, Lemei Li, Xuechao Liu, Weiwei Hao \\ College of Civil Engineering and Architecture, Shandong University of Science and Technology, Qingdao 266590, China
}

Corresponding Author Email: skd994647@sdust.edu.cn

https://doi.org/10.18280/ijsse.100206

Received: 10 November 2019

Accepted: 5 February 2020

\section{Keywords:}

urban comprehensive disaster prevention planning (UCDPP), transfer bridge, strategy generation system (SGS), two-dimensional (2D) tables

\begin{abstract}
The rapid development of big data and artificial intelligence (AI) makes it possible to make intelligent decisions on urban comprehensive disaster prevention planning (UCDPP). Based on extension problem model and transfer bridge, this paper formulates a strategy generation system (SGS) for the UCDPP, with the aid of the AI, database technology, and extension logic analysis tools. The established system consists of three layers and multiple libraries, namely, basic database, rule base (including strong correlation rules and problem rules), question base, example database, extension transform library, and strategy base. Compared with traditional SGSs, two libraries were added to our system, i.e. knowledge base and transfer bridge library, making our system more comprehensive. Taking gas station location problem in a city as an example, the feasibility of our system was confirmed, and the visual interface of the system was illustrated in details. The research results enable urban planners to make rational decisions in the process of the UCDPP.
\end{abstract}

\section{INTRODUCTION}

Urban comprehensive disaster prevention planning (UCDPP) refers to the overall design of land use and spatial layout and specific arrangement of space and facilities in a period, all of which are related to disaster prevention in urban areas [1]. During the planning, the disaster prevention facilities must be integrated into recreational facilities, and the various disaster prevention plans must be combined into an organic whole [2]. Therefore, the UCDPP is a multi-objective decision-making problem for urban planners. This problem is very difficult to solve, due to the complexity of urban functions and the uncertainty of changes brought by disasters. In most cases, the UCDPP is solved empirically by urban planners.

In the era of the Internet, many have attempted to prepare urban disaster prevention plans intelligently based on expert decision-making systems and cloud decision-making systems. However, most of these attempts only focus on a single disaster, failing to realize comprehensive disaster prevention. For instance, Fan et al. [3] suggested integrating artificial intelligence (AI) and human intelligence into disaster management. Based on building information model (BIM) and automated consistency checking, Messaoudi and Nawari [4] put forward a new disaster-resistance method called disaster resilience integrated framework for transformation (DRIFT), which bridges up the theories and practices of disaster resistance. Inspired by UN-Habitat's urban system model, Etinay et al. [5] designed a framework for disaster risk management (DRM) and disaster risk reduction (DRR), which is a risk-tolerant mechanism conducive to the sustainability of the building environment.

So far, some urban planners have tried to design urban master plans for intelligent cities, with the aid of strategy generation system (SGS) [6]. A typical SGS is the transfer bridge, a strategy to achieve the coexistence between opposing parties, which are connected or separated, under the idea of "each behaves in his/her own way and remains in his/her own place" [7]. The transfer bridge is suitable for multi-objective decision-making of the UCDPP, e.g. the integrated design of recreational facilities and disaster prevention facilities in the light of multiple disasters.

In the light of the above, this paper clarifies the basic principles and operation process of transfer bridge SGS in the UCDPP, sets up the architecture of transfer bridge SGS, and provides database support to the strategy generation using various functional modules. The proposed system stores and expresses problems, rules, models, and strategies as twodimensional (2D) data tables. Specifically, the extension problem model and the transfer bridge method were used to build and express the problem model, and formulate the preliminary strategy. The strategy was verified and improved by expert knowledge. The proposed system enables urban planners to make reasonable UCDPP decisions that are highly applicable and intelligent.

\section{PRELIMINARIES}

In this paper, the UCDPP problem is collected and classified automatically by the extension problem model and correlation functions. Then, the objectives and constraints of the problems were identified. Through extension analysis and extension transform, the connected transfer part and the separated transfer part were constructed, forming the preliminary strategy. Then, the transfer bridge was verified by the correlation functions in the rule base, and the planning results of the strategy were expressed. Finally, multiple strategies 
were optimized through evaluation.

\subsection{Problem modelling}

The UCDPP problem was modelled by the extension problem model, and divided into two parts: the objectives and the constraints. Then, the objective set and constraint set were unified as the primitives. Therefore, the problem can be expressed as $(G 1 \wedge G 2 \wedge \ldots \wedge G n) \uparrow L$, where $G_{i}$ is an objective, $\mathrm{L}$ is the constraint set, $\Lambda$ is the extension operation, and $\uparrow$ is disjunction.

Besides artificial analysis, the problem was also automatically modelled by judging the coexistence degree between goals and limits against the pre-set rule base. In this way, new problems were automatically detected during the generation of UCDPP strategies, making the decisions on UCDPP more comprehensive and compatible to complex disaster constraints.

\subsection{Generation of transfer bridge}

Compared with single-objective SGSs, transfer bridge SGS not only pursues the primary goal, but also considers secondary goals. In this paper, the transfer bridge is constructed based on the extension SGS. First, the rules of extension analysis were determined based on the question base and rule base. Then, the multi-disaster UCDPP problem was converted into conditional transform channels, and the integrated design between recreational facilities and disaster prevention facilities was converted into objective transform channels. After that, the rules of extension transform from the knowledge base were adopted to form the connected transfer part and the separated transfer part. On this basis, the initial strategy was developed as the input of the strategy generation stage, and subjected to evaluation and verification.

\subsection{Strategy generation}

The UCDPP strategies were generated in four steps: verification, evaluation, storage, and result expression. Overall, the UCDPP strategies are created based on transfer bridge according to the workflow in Figure 1.

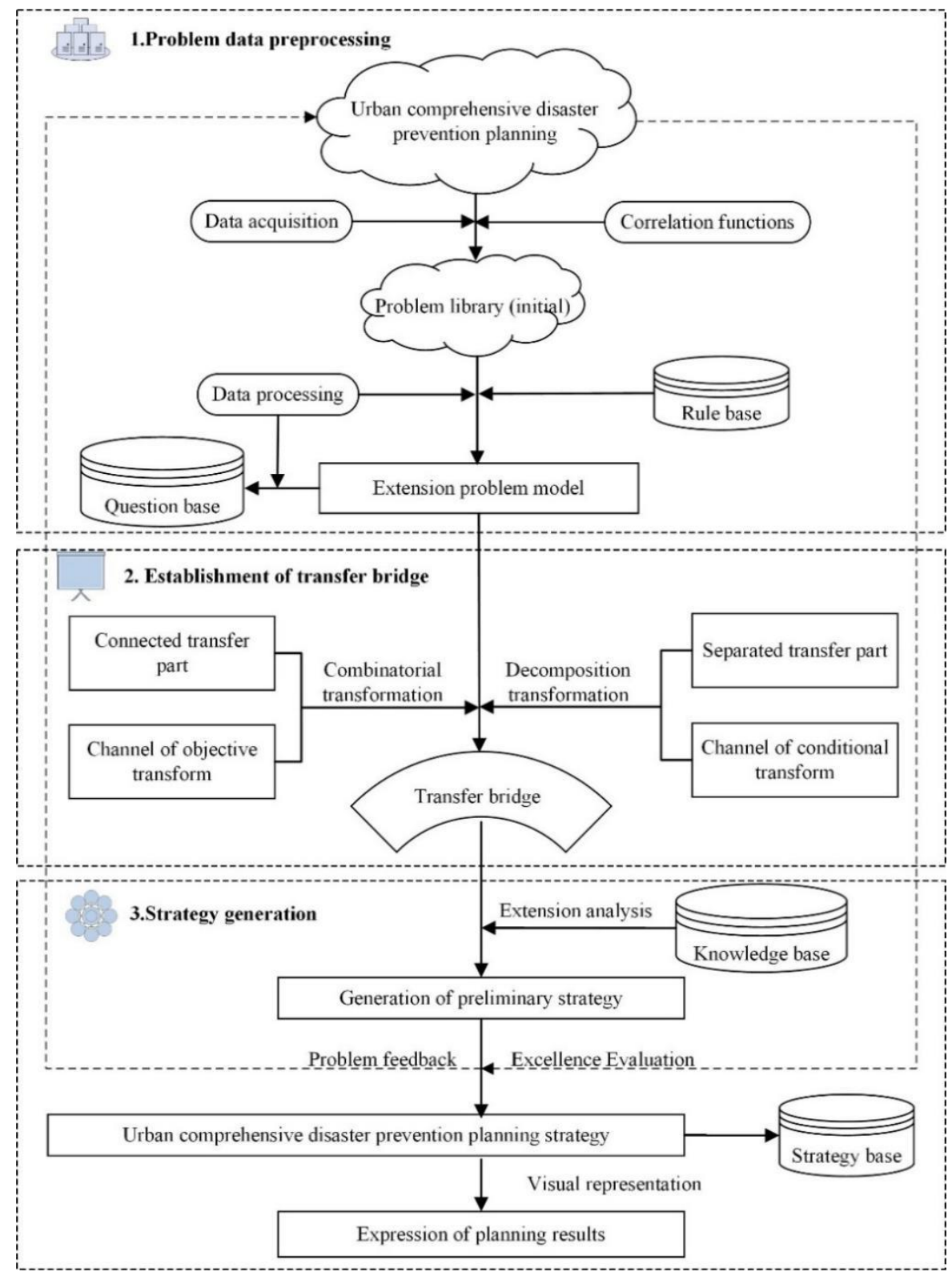

Figure 1. The workflow of the generation of UCDPP strategies based on transfer bridge 


\section{ARCHITECTURE OF TRANSFER BRIDGE SGS}

Based on the above workflow, this paper establishes a logical model for the transfer bridge SGS for the UCDPP. The logical model has three layers and multiple libraries.

The basic data layer provides the classification data required for system operation, including question base, rule base, knowledge base, and strategy base.

The logic analysis layer is the middle layer of the model. There are four subsystems on this layer: the question base subsystem, the rule base subsystem, the knowledge base subsystem, and the strategy base subsystem. The couplings between these subsystems are provided for extension analysis and logic judgment. Besides, the subsystems are also managed through addition, deletion, modification, and examination.

The human-computer interaction (HCI) layer is the top layer of the model. This layer is responsible for the interaction between user and system, including problem input, rule selection, and strategy output.

The software structure of the transfer bridge SGS for the UCDPP was developed on the model-view-controller (MVC) pattern [8], and realized by techniques like Servlet, Enterprise Java Bean (EJB), and Java Sever Pages (JSP). Specifically, the application representation was achieved by the JSP, where the foreground service is provided by AngularJS and the application web is provided by Servlet Service process control [9]. The specific business modules were implemented by EJB [10], the database-related operations were performed on object relational mapping (ORM) [11], identity authentication and authorization were realized by JSON Web Token (JWT) [12], and data persistence was enabled by MySQL database [13], and the three-tier structure of the system is as shown in Figure 2.

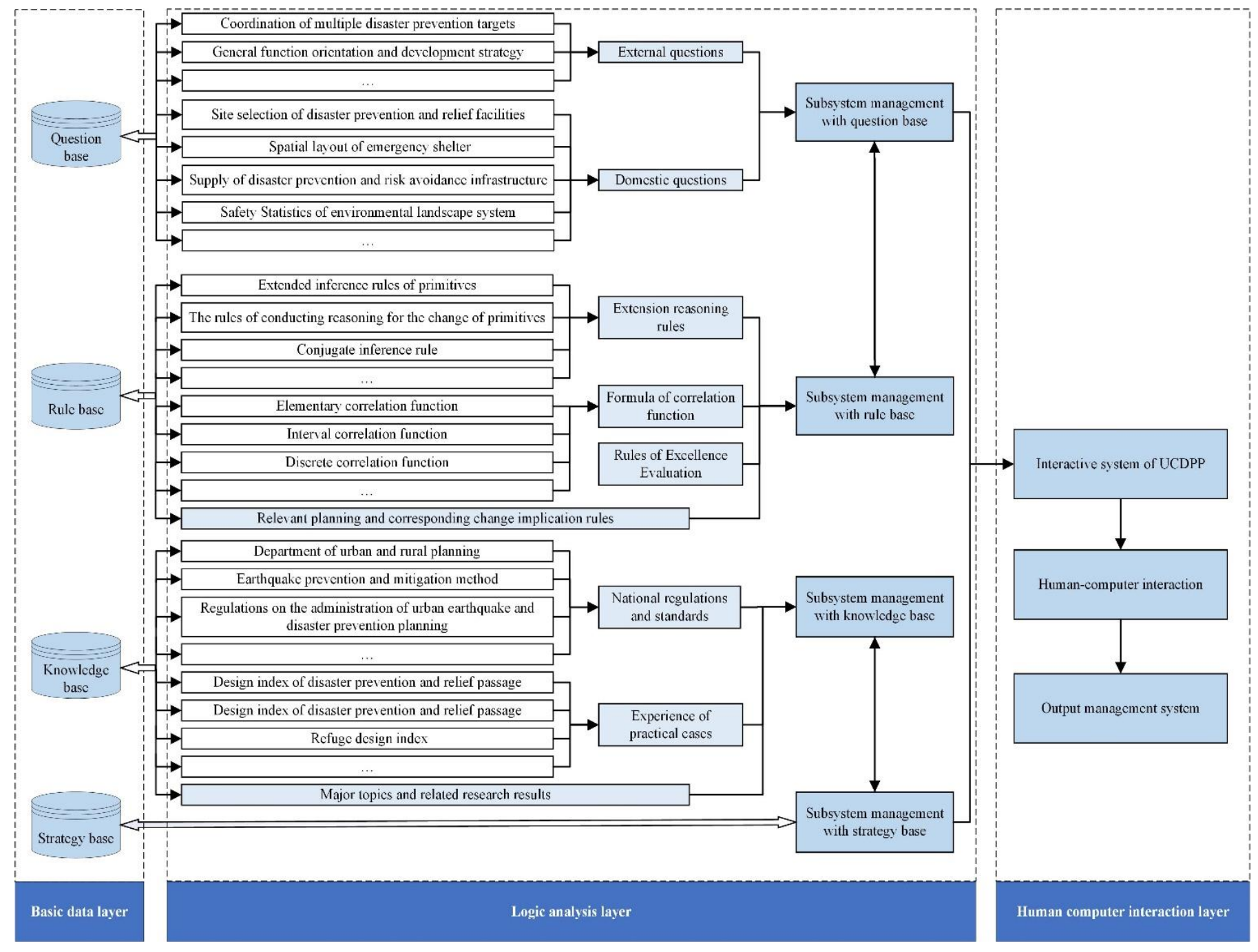

Figure 2. The architecture of the transfer bridge SGS for the UCDPP

\section{DESIGN OF DATABASE OPERATION MODEL}

Different kinds of multidimensional data were converted into $2 \mathrm{D}$ tables, using relational database. In the relational database, the objectives, constraints, transform method, transfer bridge, and strategies of the UCDPP are all expressed as primitives, and imported to the corresponding libraries as 2D tables.
Based on the types of data, the following libraries are generally included in extension SGS, namely, basic database, rule base (including strong correlation rules and problem rules), question base, example database, extension transform library, and strategy base. Two libraries were added to our system, i.e. knowledge base and transfer bridge library, making our system more comprehensive. 
This section takes question base, extension transform library, transfer bridge library, and strategy base as examples to explain the design of database operation model.

\subsection{Design of question base}

The problems of UCDPP were collected preliminarily by Natch software. Then, correlation functions were introduced to screen and classify the collected problems based on data types, forming the initial question base for the UCDPP.
The rule base was added to set the rules for data processing of Navicat software, facilitating the derivation of the extension problem model. The data processing optimizes the UCDPP data, resulting in a complete question base of UCDPP.

The core elements of problem data are objectives and constraints, which are deeply correlated with each other. These primitives were converted into $2 \mathrm{D}$ data tables for storage, analysis, and calculation. Table 1 shows the $2 \mathrm{D}$ table converted from the correlation between an objective and a constraint.

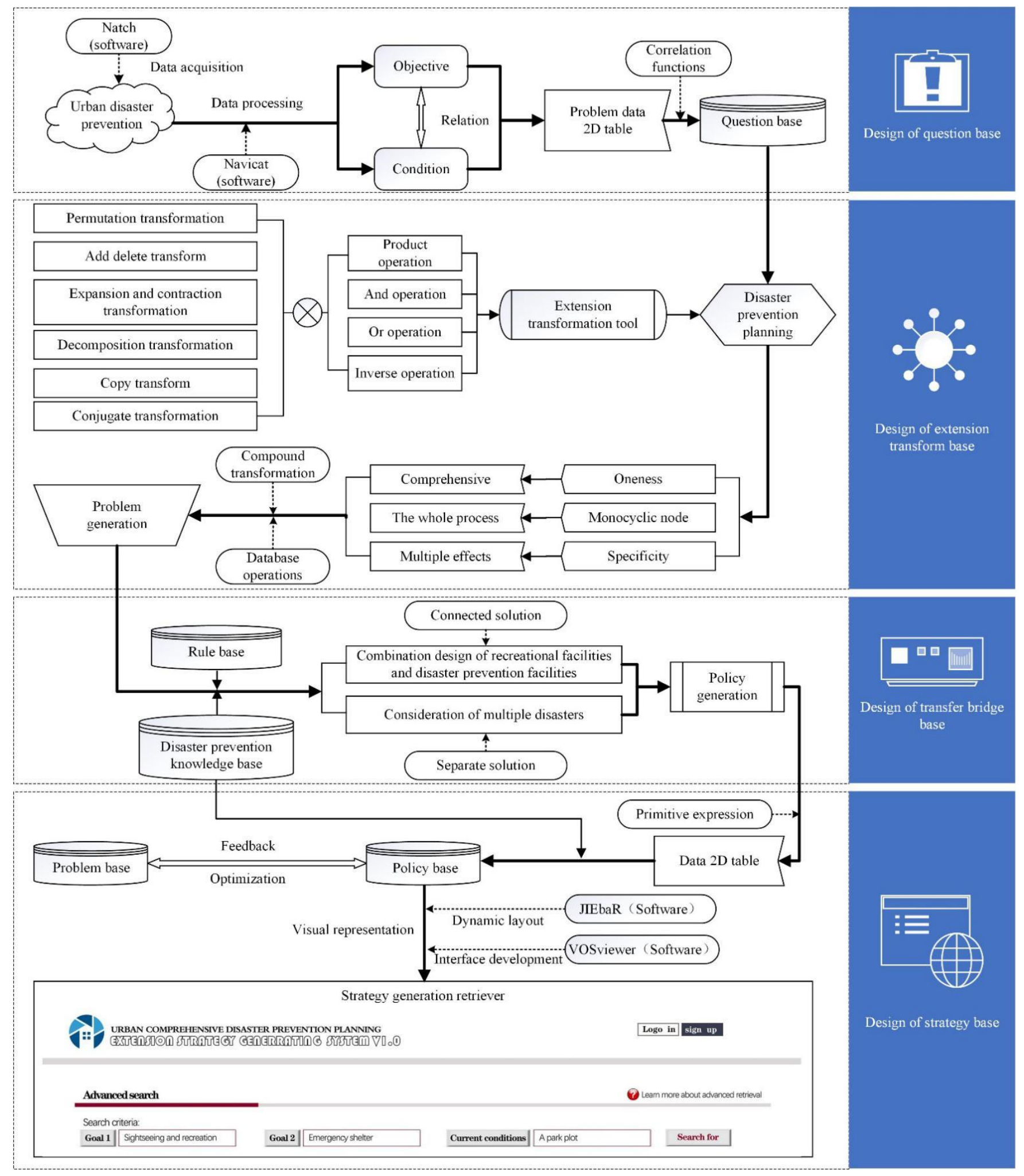

Figure 3. The workflow of the database operation model 
Table 1. The 2D table for the correlation between an objective and a constraint

\begin{tabular}{ccc}
\hline Objective code & Constraint code & Correlation code \\
\hline$G_{i}$ & $L_{j}$ & $A_{i j}$ \\
\hline
\end{tabular}

\subsection{Design of extension transform library}

Let $d, b$, and $u$ be the object, feature, and feature magnitude of the extension transform tool, respectively. Then, the extension transform tool can be expressed as:

$$
\begin{aligned}
& T=\left[\begin{array}{ccc}
d & b_{1} & u_{1} \\
& \ldots & \ldots \\
& b_{n} & u_{n}
\end{array}\right]= \\
& \text { [transformation, planning carrier, disaster prevention park } \\
& \text { planning type, detailed design } \\
& \text { planning means, combination of disaster relief }
\end{aligned}
$$

The transform types include displacement, addition and deletion, expansion and contraction, decomposition, copy, and conjugation. To deal with the complex problems in UCDPP, many other transform methods could be obtained by superposing existing computing methods. Our extension transform library contains four operations: product operation $T_{1} T_{2}$, and operation $T_{1} \wedge T_{2}$, or operation $T_{I} \vee T_{2}$, and inverse operation $\bar{T}$.

Product operation solves contradictory problems through two or more transforms in a row: $T=T_{n-1} T_{n-2} \ldots T_{2} T_{1}$;

And operation solves contradictory problems through two or more simultaneous transforms: $T=T_{1} \wedge T_{2} \wedge T_{3} \wedge \ldots \wedge T_{n}$;

Or operation solves contradictory problems by choosing one out of many transforms: $T=T_{1} \vee T_{2} \vee T_{3} \vee \ldots \vee T_{n}$;

Inverse operation solves contradictory problems by choosing one or more of the transforms: For objective $M$, there is a transform $T$ to make $T M=M^{\prime}$; For transform $T$, make $T^{\prime} M^{\prime}$ $=M ;$ then, $T=T^{-1}$.

Through the above operations, it is possible to solve the transform of UCDPP problems in a comprehensive manner. Here, the transform methods are combined with the database operation technology to quickly solve the problems in UCDPP, which involve multiple influencing factors.

\subsection{Design of transfer bridge library}

The transfer bridge can be solved in two ways: connected type and separated type. The former is more suitable to solve multiple disasters, and the latter is more suitable to solve the integrated design between recreational facilities and disaster prevention facilities. The analysis and storage of the transfer bridge are essential to the SGS. The elements in the transfer bridge library should correspond to those in the question base one by one. The generation process can be described by the feature properties.

\subsection{Design of strategy base}

Once generated, UCDPP strategies were sorted dynamically by jiebaR software and stored in the strategy base. Then, the VOSviewer software was adopted to develop the retravel interface. The entire database operation model is displayed in Figure 3 above.

\section{EXAMPLE ANALYSIS}

To verify its effectiveness, the proposed transfer bridge SGS was applied to solve the UCDPP of a city.

\subsection{Problem generation}

In the target city, one gas station needs to be built along the main road, in order to make up for the coverage gap of gas stations. However, it is difficult to find the construction land that satisfy the requirements of all aspects, under the limits of safe distance and service radius. The contradiction of site selection is explained in Figure 4.

Then, the extension problem model was established: the current constraint $L$ cannot meet the objectives $G_{1}$ and $G_{2}$, i.e. $(G 1 \wedge G 2) \uparrow L$

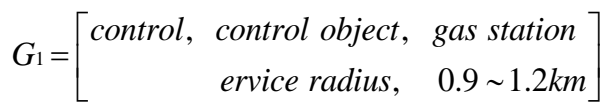

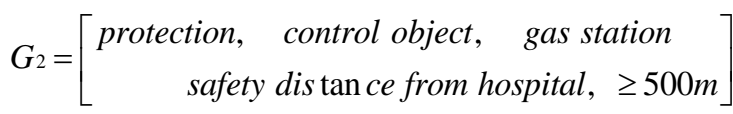

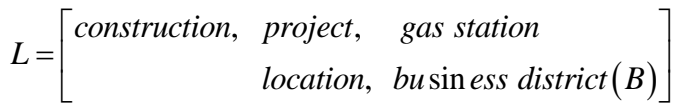

According to the question base subsystem, the objectives and constraint of the problem were converted into 2D data tables, and stored in the objective database and constraint database of the question base, respectively (Tables 2, 3 and 4).

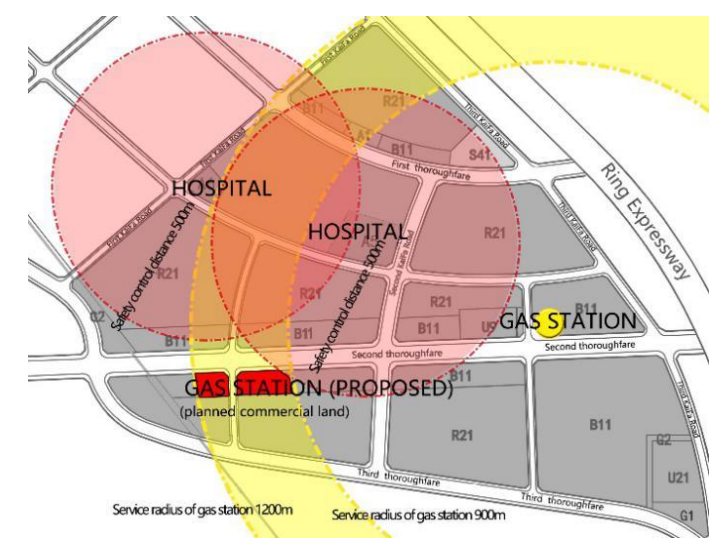

Figure 4. The constraints on the location of the gas station

Table 2. The 2D table of the common objective of gas station location

\begin{tabular}{cccc}
\hline $\begin{array}{c}\text { Objective } \\
\text { code }\end{array}$ & Object & $\begin{array}{c}\text { Planning } \\
\text { function }\end{array}$ & Location requirement \\
\hline $\mathrm{G}_{1}$ & Gas station & Fuel service & Service radius: $0.9-1.2 \mathrm{~km}$ \\
\hline
\end{tabular}

Table 3. The 2D table of the disaster prevention objective of gas station location

\begin{tabular}{cccc}
\hline $\begin{array}{c}\text { Objective } \\
\text { code }\end{array}$ & Object & $\begin{array}{c}\text { Planning } \\
\text { function }\end{array}$ & $\begin{array}{c}\text { Location } \\
\text { requirement }\end{array}$ \\
\hline $\mathrm{G}_{2}$ & Gas station & $\begin{array}{c}\text { Explosion risk } \\
\text { control }\end{array}$ & $\begin{array}{c}\text { safety distance with } \\
\text { hospital: } \geq 500 \mathrm{~m}\end{array}$ \\
\hline
\end{tabular}


Table 4. The 2D table of the constraint of gas station location

\begin{tabular}{cccc}
\hline Constraint code & Object & Site location & Area \\
\hline $\mathrm{L}_{1}$ & Gas station & Business district & $2,000 \mathrm{~m}^{2}$ \\
\hline
\end{tabular}

\subsection{Generation of transfer bridge}

Location planning is the opposite of the integrated design between recreational facilities and disaster prevention facilities.

Therefore, the implicative channel of objectives should be built to generate the transfer bridge. The rules of correlation transform were expressed as 2D tables (Table 5).

Besides, some special land use functions are compatible with gas stations. For example, Suqian, Jiangsu tried to integrate gas station layout with other municipal facilities, aiming to meet the various requirements on gas station location.

Here, the transfer bridge of the location planning problem can be established as:

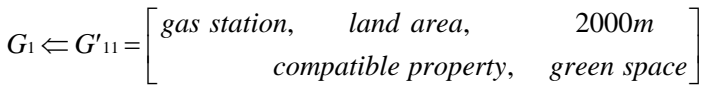

$$
\begin{aligned}
& \left(G^{\prime}{ }_{111}=(\text { gas station, } \text { compatible nature, } \text { street view })\right. \\
& G^{\prime}{ }_{112}=(\text { gas station, compatible nature, street view }) \\
& \dashv\left\{G_{113}^{\prime}=(\text { gas station, compatible nature, waste transfer station })\right. \\
& G^{\prime}{ }_{114}=(\text { gas station, compatible nature, retail small bu } \sin \text { ess }) \\
& G^{\prime}{ }_{114}=(\text { gas station, compatible nature, fire station })
\end{aligned}
$$

$$
\begin{gathered}
G_{2} \Leftarrow G^{\prime}{ }_{21}=\left[\begin{array}{rr}
\text { isolation, } & \text { object, } \\
\text { width, } & \text { hospital } \\
\text { wo0m }
\end{array}\right] \\
G^{\prime}{ }_{113}=G_{1} \otimes Z_{1} ; G^{\prime}{ }_{21}=G_{2} \otimes Z_{2}
\end{gathered}
$$

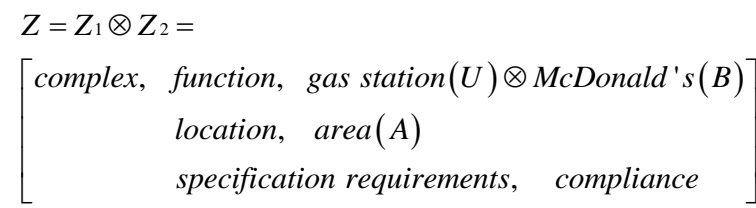

Table 5. The 2D tables of the transition bridge for gas station location

\begin{tabular}{cccccc}
\hline Question code & Transform type & Transform object & Transfer part 1 & Transfer part 2 \\
\hline Key & Interconnection & Land compatibility & Business land (B) & Utility land (U) \\
\hline
\end{tabular}

\subsection{Strategy verification and optimization}

According to relevant regulations, the fire safety distance between gas stations and important buildings should be greater than or equal to $50 \mathrm{~m}$; the service radius of gas stations in urban areas should be controlled within $0.9-1.2 \mathrm{~km}$. These regulations can be transformed into primitives as:

$$
\begin{aligned}
& Z=Z_{1} \otimes Z_{2}= \\
& {\left[\begin{array}{c}
\text { complex, combined function, gas station } \otimes \text { commercial land } \\
\text { dis } \tan \text { ce from nearest gas station, } 2 \mathrm{~km} \\
\text { dis } \tan \text { ce from municipal hospital, } 60 \mathrm{~m}
\end{array}\right]}
\end{aligned}
$$

Taking the safety distance $(50 \mathrm{~m})$ as the minimum critical condition, the discrete correlation function can be established as:

$$
K_{1}(X)=\left\{\begin{array}{l}
1, x \geq 50 m \\
0, x<50 m
\end{array}\right.
$$

In terms of service radius, the control distance is $0.9-1.2 \mathrm{~km}$. Through double conversion, the distance between adjacent gas stations should be $1.8-2.4 \mathrm{~km}$. Hence, the interval correlation function can be set up:

$$
K_{2}(X)=\left\{\begin{array}{l}
\frac{x-1.8}{2.4-1.8}, x<1.8 \mathrm{~km} \\
\frac{2.4-x}{2.4-1.8}, x \geq 1.8 \mathrm{~km}
\end{array}\right.
$$

Finally, the coexistence degree of the transfer bridge (Table 6) was judged to see whether it can solve the gas station location problem. The coexistence degree $K=K_{1} * K_{1}=0.67>0$, indicating that the transfer bridge provides a suitable solution to the said problem.

Table 6. The 2D table of the strategy for gas station location

\begin{tabular}{ccccc}
\hline $\begin{array}{c}\text { Strategy ID } \\
\text { Key }\end{array}$ & $\begin{array}{c}\text { Planning type } \\
\text { Hazardous site }\end{array}$ & $\begin{array}{c}\text { Disaster prevention carrier 1 } \\
\text { Gas station }\end{array}$ & $\begin{array}{c}\text { Disaster prevention carrier 2 } \\
\text { Municipal hospital }\end{array}$ & $\begin{array}{c}\text { Transfer bridge type } \\
\text { Interconnection }\end{array}$ \\
\hline $\begin{array}{c}\text { Implementation } \\
\text { object }\end{array}$ & $\begin{array}{c}\text { Implementation } \\
\text { feature }\end{array}$ & $\begin{array}{c}\text { Implementation } \\
\text { value }\end{array}$ & $\begin{array}{c}\text { Coexistence } \\
\text { degree }\end{array}$ & $\ldots$ \\
Land usage & Compatibility & Double stack & 0.67 & $\ldots$ \\
\hline
\end{tabular}

\section{VISUAL INTERFACE DESIGN}

As shown in Figure 5, the visual interface of the transfer bridge SGS covers four parts: query condition setting area, operation method setting area, display mode setting area, and query result sorting area.
(1) Query condition setting area

Objectives 1 and 2 and constraint $L$ were inputted to describe the contradiction in the UCDPP. The two objectives could be inputted separately to illustrate the and contradiction. 


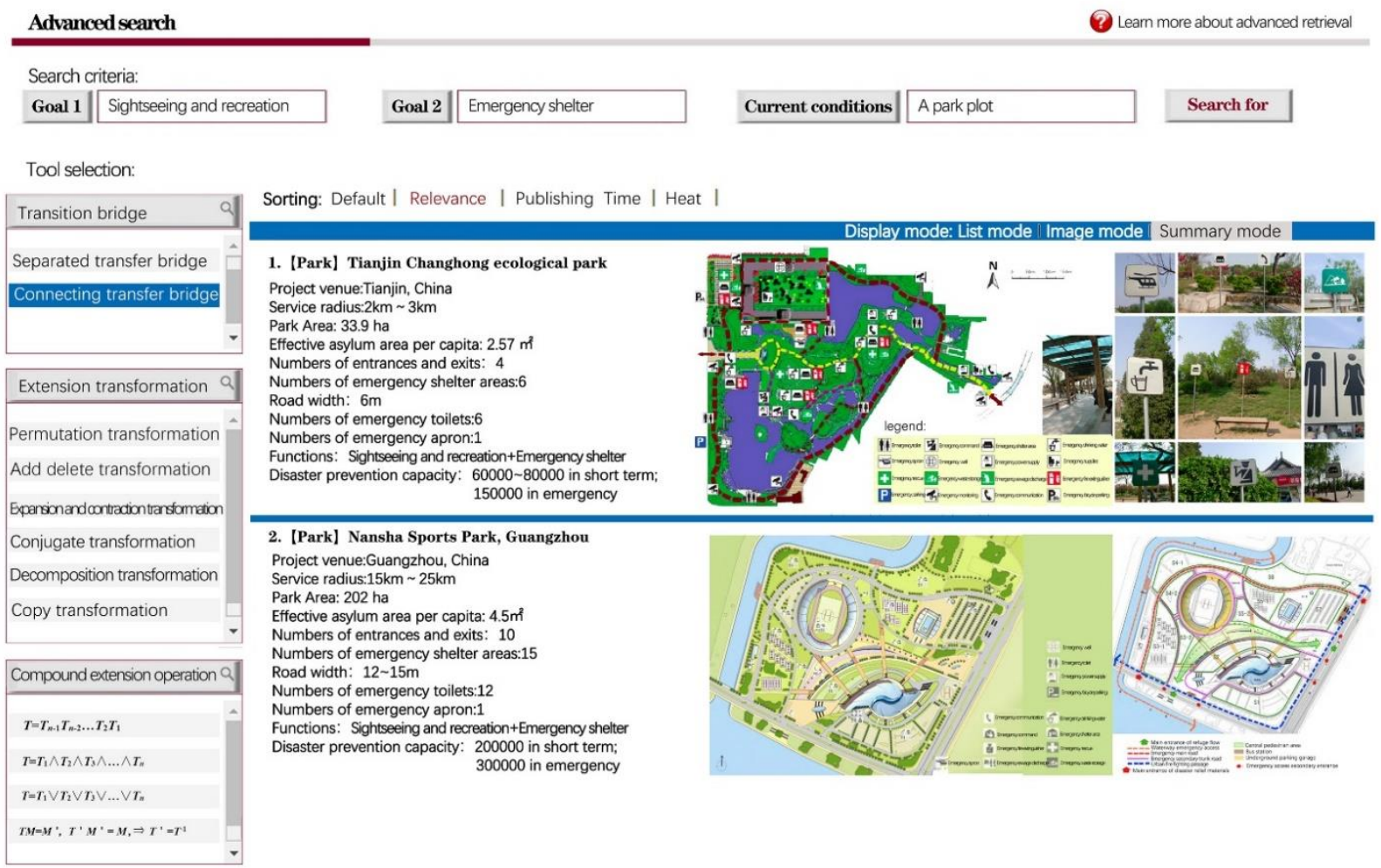

Figure 5. The visual interface of the transfer bridge SGS for the UCDPP

(2) Operation method setting area

The extension type and tools were selected in this area. First, the transfer bridge type, separation type, and connection type were chosen. Then, the basic transforms were selected, namely, permutation, addition and deletion, expansion and contraction, decomposition, replication, and conjugation. Finally, the operations of extension transform were identified, including composite operation, product operation, and operation, or operation, and reverse operation.

(3) Display mode setting area

This area supports quick and advanced queries based on strategy relevance, innovation degree, and other features. The strategy is presented in the form of basic data, text summaries, and design drawings.

(4) Query result sorting area
There are three display modes: strategy list preview, strategy summary preview, and thumbnail preview. Take the integrated design between recreational facilities and disaster prevention facilities for example. Once the query conditions and calculation methods were set, the corresponding strategy was displayed in the summary preview.

\section{DISCUSSION}

As shown in Figure 6, the proposed transfer bridge SGS is capable of solving two types of disaster prevention planning problems, namely, the integrated design between recreational facilities and disaster prevention facilities, and the planning based on multiple disasters. The two types of problems are classified based on the root cause of the objective conflict.

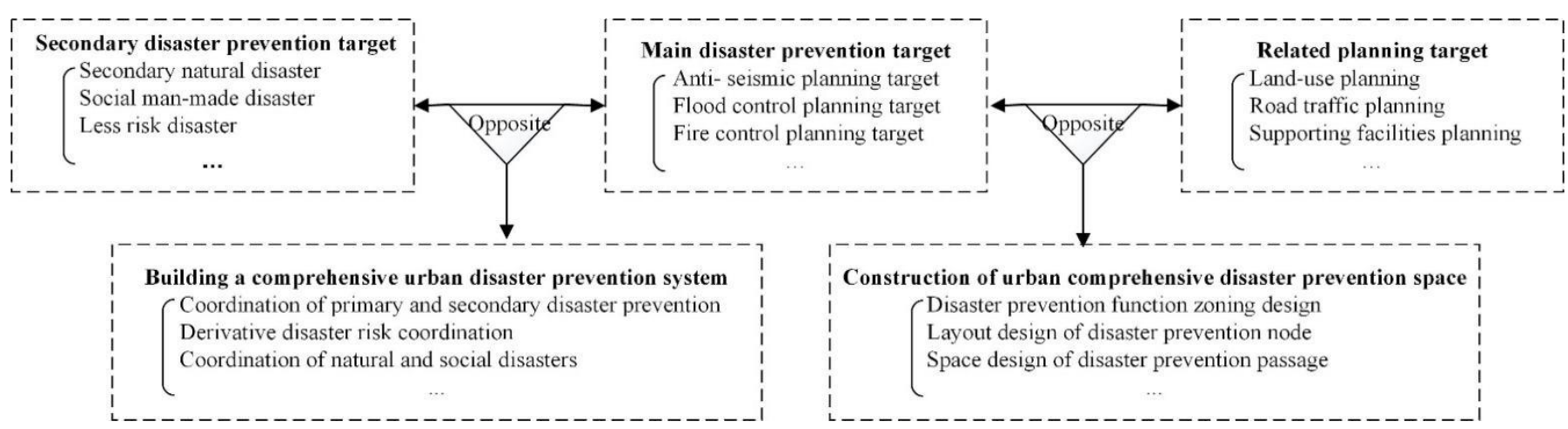

Figure 6. The application scope of the transfer bridge SGS for the UCDPP 
According to the time factor, the integrated design between recreational facilities and disaster prevention facilities can be divided into peacetime problem and disaster time problem. According to the types of disasters, the multi-disaster planning can be split into the planning under primary and secondary disasters and the planning under multiple types of derived disasters

Besides solving disaster prevention problem at the level of detailed design, the transfer bridge SGS for the UCDPP can also help generate decisions at the level of master planning according to the main type of disasters determined through risk assessment, making it easy to build the spatial pattern of the UCDPP system.

\section{CONCLUSIONS}

This paper firstly introduces the basic principles of extension transfer bridge method. Then, the framework of the transfer bridge SGS was created for the UCDPP, followed by the construction of database operation model for the system. After that, the visual interface and application scope of our method were detailed. Overall, our system was developed based on transfer bridge method, decision system architecture, and database technology. Finally, the feasibility of our system was confirmed through example analysis. Compared with existing SGSs, our system enables urban planners to make reasonable UCDPP decisions that are highly applicable and intelligent. The future research will further improve the software design of the proposed system.

\section{REFERENCES}

[1] Dai S.Z. (2011). Urban Comprehensive Disaster Prevention Planning. China Architecture \& Building Press, 32-33.

[2] Wang, J.B. (2007). A study on planning methods of urban comprehensive disaster prevention plan in China. Planners, 23(1): 53-95. http://dx.doi.org/10.3969/j.issn.1006-0022.2007.01.015.

[3] Fan, C., Zhang, C., Yahja, A., Mostafavi, A. (2019).
Disaster city digital twin: A vision for integrating artificial and human intelligence for disaster management. International Journal of Information Management, 102049. https://doi.org/10.1016/j.ijinfomgt.2019.102049

[4] Messaoudi, M., Nawari, N.O. (2020). BIM-based Virtual Permitting Framework (VPF) for post-disaster recovery and rebuilding in the state of Florida. International Journal of Disaster Risk Reduction, 42: 101349. https://doi.org/10.1016/j.ijdrr.2019.101349

[5] Etinay, N., Egbu, C., Murray, V. (2018). Building urban resilience for disaster risk management and disaster risk reduction. Procedia Engineering, 212: 575-582. https://doi.org/10.1016/j.proeng.2018.01.074

[6] Li, W.K. (2011). Study on decision making of extension urban planning. School of architecture, Harbin University of Technology, Harbin Institute of Technology.

[7] Cai, W., Yang, C.Y. (2014). Extenics. Science Press, 228 229.

[8] Hannemann, J., Kiczales, G. (2002). Design pattern implementation in Java and AspectJ. In Proceedings of the 17th ACM SIGPLAN Conference on ObjectOriented Programming, Systems, Languages, and Applications, pp. 161-173. https://doi.org/10.1145/583854.582436

[9] Richardson, L., Ruby, S. (2008). RESTful Web Services. O'Reilly Media Inc, 42-58.

[10] Guamán, D., Guamán, F., Jaramillo, D., Correa, R. (2016). Implementation of techniques, standards and safety recommendations to prevent XSS and SQL injection attacks in Java EE RESTful applications. In New Advances in Information Systems and Technologies, 444(3): 691-706. https://doi.org/10.1007/978-3-31931232-3_65

[11] Kopteff, M. (2008). The usage and performance of object databases compared with ORM tools in a java environment. In ICOODB, 199-218.

[12] Jones, M.B. (2011). The emerging JSON-based identity protocol suite. In $\mathrm{W} 3 \mathrm{C}$ Workshop on Identity in the Browser, pp. 1-3.

[13] Matthews, M., Cole, J., Gradecki, J.D. (2003). MySQL and Java Developer's Guide. John Wiley \& Sons, 35-68. 